\title{
What makes working memory spans so predictive of high-level cognition?
}

\author{
RAPHAËLLE LÉPINE and PIERRE BARROUILLET \\ Université de Bourgogne, Dijon, France \\ and \\ VALÉRIE CAMOS \\ Université René Descartes-Paris 5, Paris, France
}

\begin{abstract}
Working memory (WM) span tasks involving a complex activity performed concurrently with item retention have proven to be good predictors of high-level cognitive performance. The present study demonstrates that replacing these complex self-paced activities with simpler but computer-paced processes, such as reading successive letters, yields more predictive WM span measures. This finding suggests that WM span tasks evaluate a fundamental capacity that underpins complex as well as elementary cognitive processes. Moreover, the higher predictive power of computer-paced WM span tasks suggests that strategic factors do not contribute to the relationship between WM spans and high-level cognition.
\end{abstract}

The theoretical construct of working memory has played an increasingly important role in accounting for cognition, and especially for high-level cognition. Working memory (WM) usually refers to a cognitive system devoted to the simultaneous maintenance and treatment of information that is involved in the planning, coordination, and control of high-level cognitive processes (Baddeley \& Hitch, 1974; Daneman \& Carpenter, 1980). This central role in cognition led many authors to propose span tasks that aimed at assessing WM capacity in individuals. In contrast to traditional short-term memory span tasks, which require simple maintenance and recall of information such as digits, letters, or words, WM span tasks involve a processing component in addition to the maintenance of to-be-recalled items. For example, individuals are asked to read sentences while maintaining their final words (reading span; Daneman \& Carpenter, 1980) or to solve arithmetic problems while maintaining words (operation span; Turner \& Engle, 1989).

These WM span measures proved to reliably predict, better than short-term memory spans, performance in a wide range of complex activities such as reading comprehension (Daneman \& Carpenter, 1980; Daneman \& Merikle, 1996), complex learning (Shute, 1991), and reasoning (Barrouillet, 1996; Kyllonen \& Christal, 1990). Moreover, it has been demonstrated that, in contrast to short-term memory, WM shows a strong connection to

This work was presented at the Thirteenth Conference of the European Society of Cognitive Psychology in Granada, Spain, September 2003. The authors thank Karine Petetin for collecting the data. Correspondence concerning this article should be addressed to R. Lépine, Université de Bourgogne, LEAD-CNRS 5022, Pôle AAFE, Esplanade Erasme, B.P. 2651321065, Dijon, France (e-mail: raphaelle.lepine@leadserv.u-bourgogne.fr). fluid intelligence (Engle, Tuholski, Laughlin, \& Conway, 1999). In order to account for these relationships, it has been proposed that WM measures assess a fundamental capacity required by complex activities, which is conceived of as a capacity to control attention (Engle, Kane, \& Tuholski, 1999) or to supervise and coordinate multiple-system functioning (Baddeley, 1990). Consequently, the activities included as processing components in WM span tasks are usually selected from those thought to require a high level of executive control (e.g., problem solving, reading comprehension, reasoning, mental calculation). The underlying idea is that more controlled and complex activities provide better WM span measures because complex activities tap the limited pool of cognitive resources sufficiently to disrupt maintenance and permit an accurate measure of WM capacity.

However, is the complexity of the processing component of WM span tasks necessary to disrupt maintenance and accurately assess WM capacity? Barrouillet, Bernardin, and Camos (2004) have recently shown that very simple activities included as processing components in WM span tasks have an equally detrimental effect on recall as do complex activities, provided that they are not self-paced but computer-paced. Remembering letters while solving running operations such as adding or subtracting 1 to or from digits, or even merely reading digits successively presented on a screen at a fast pace, was very difficult, and adult participants exhibited WM spans lower than 3 in these tasks. The authors accounted for this effect by proposing that simple but time-constrained activities capture a sufficient amount of attention to disrupt the maintenance of items to be recalled.

Nonetheless, the value of the WM span tasks is to predict performance on complex cognitive activities. Al- 
though the new tasks designed by Barrouillet et al. (2004) have the expected detrimental effect on recall, it remains to be established whether they assess the same capacity and have the same predictive power as traditional WM span tasks. Indeed, two alternative hypotheses can be put forward to account for the well-known relationship between WM span and performance in complex cognitive activities. According to the first hypothesis, traditional WM tasks would evaluate some general cognitive capacity, described by Barrouillet et al. as an attentional capacity, that is involved in any cognitive process requiring access and maintenance of items of knowledge. This limited attentional capacity would thus underpin and constrain each step of the complex activities involved in high-level cognition. According to this view, complexity is an unnecessary characteristic of the processing component of the WM tasks because what is required is an activity that captures attention. Moreover, the temporal constraints of Barrouillet et al.'s computer-paced WM tasks hamper the use of possible strategies for coping with the specific demands of the dual-task paradigmstrategies that are allowed by most of the traditional selfpaced WM span tasks (Baddeley, Logie, Nimmo-Smith, \& Brereton, 1985; Case, Kurland, \& Goldberg, 1982; Daneman \& Carpenter, 1980; Turner \& Engle, 1989). ${ }^{1}$ According to the fundamental capacity hypothesis, these strategies may produce biased measures of the fundamental capacity. Thus, this hypothesis predicts that the spans provided by the new tasks will be more predictive of performance in high-level cognition than the traditional tasks are. An alternative hypothesis would be that the traditional tasks help to evaluate some high-level executive capability that is essential both for the complex processes that they involve and for the use of strategies for dealing with the requirements of the dual task. HighWM-span individuals would be those who are better able to strategically plan and monitor their activity in complex situations, achieving better performance in WM dual tasks and in higher level cognition. According to this account, WM spans are predictive because the traditional tasks mimic high-level cognitive activities. This hypothesis predicts that the traditional spans will have a greater predictive value because the new tasks involve fewer strategic factors and only elementary processes.

We tested these two hypotheses by comparing the correlation between the academic achievements of 11-yearold children on the one hand, and WM spans evaluated either by traditional or by new WM span tasks on the other. The traditional WM tasks were a reading span task, inspired by Baddeley et al. (1985), in which participants had to read and understand sentences, and an operation span task, inspired by Turner and Engle (1989), in which participants verified complex equations. The new tasks were (1) a reading letter span task in which, while maintaining digits, the children had only to read letters instead of understanding sentences, and (2) the continuous operation span task of Barrouillet et al. (2004), in which, while maintaining letters, the children performed simple operations [i.e., addition (subtraction) of 1 to (from) numbers from 1 to 9], in contrast to the complex equations of the operation span task.

\section{METHOD}

\section{Participants}

Ninety-three French sixth graders attending a suburban public middle school (43 girls and 50 boys; mean age $=11$ years, 11 months; $S D=8$ months) participated as volunteers.

\section{Materials and Procedure}

Scholastic evaluation. The scholastic evaluation was provided by individual scores from the national academic achievement test that each French sixth grader takes at the beginning of the academic year. This test yields compound scores in literacy and mathematics as well as a global scholastic score in terms of percentage of success. The literacy assessment involves language comprehension, language tools, and written production. The mathematics assessment involves geometry, number knowledge and writing of numbers, arithmetic problem solving, word problem solving, and comprehension of mathematical tools and concepts. ${ }^{2}$

Traditional working memory span tasks. In the reading span task, the participants were asked to read aloud sentences containing 4-11 words $(M=6.9)$ while maintaining to-be-remembered numbers. These sentences were presented in sets of increasing length (from 1 to 7) with three sets of each length. Half of the 84 sentences were true, and the other half were false (e.g., "A cow lays eggs"). The participants were asked to evaluate the truthfulness of each sentence by pressing labeled keys on the keyboard. The to-beremembered numbers were randomly drawn from between 1 and 16, with the exception of 14 because it is bisyllabic in French; one number was presented before each sentence. Each set of sentences started with a 1-sec "ready" signal. After $500 \mathrm{msec}$, a first number to be remembered was displayed on the screen for $1,500 \mathrm{msec}$ and was followed by a sentence to be read after a delay of $500 \mathrm{msec}$. The children read the sentence at their own pace. When they responded on the keyboard, the sentence was deleted and, after a 500-msec delay, a 1,500-msec display of a new number was triggered. This was followed by a new sentence to be read after a 500-msec delay, and so on. At the end of the series, the word RAPPEL ("recall") was displayed on the screen and the participant had to recall the presented numbers in the correct order.

The operation span task had the same structure as the reading span task, except that sentences were replaced by equations to be verified and numbers were replaced by consonants to be remembered; each equation to be verified was preceded by a consonant to be remembered. The equations were three-operand additive problems (e.g., $6+7+2=13$ ?), the proposed result being correct for half of the problems and false for the others. The participants had to read each equation aloud before solving it. All consonants were used except W, which is trisyllabic in French.

The new working memory span tasks. The new tasks had exactly the same structure as the traditional tasks except that the processing component was computer paced and time constrained. As in the reading span task described above, in the reading letter span task the participants were presented with numbers to be remembered. Instead of sentences, sets of 4-6 letters, to be read aloud, appeared successively on the screen after each number to be remembered. These letters were randomly drawn from the 26 letters of the alphabet, provided that a given set contained neither the same letter twice nor two successive letters of the alphabet. Each letter remained on the screen for $1,000 \mathrm{msec}$ after a delay of $350 \mathrm{msec}$.

The continuous operation span task was similar to the traditional operation span task. The participants were presented with series of letters to be remembered, but the equations were replaced with run- 
ning operations. After each to-be-remembered letter, a digit, called the root, appeared for $1,500 \mathrm{msec}$, followed by a series of two, three, or four successive screens displaying either " +1 " or " -1 ." The sets of operations were randomly constructed with the constraint that no final or intermediate result was below 1 or above 9 (e.g., $8 /+1 /+1$ ). Each operation (i.e., +1 or -1 ) remained on the screen for 1,500 msec after a 500-msec delay. The participants were asked to read all the stimuli and to perform the operations aloud (e.g., for the series $5 /+1 /-1$, the participants were to utter "five, plus one, six, minus one, five").

For each WM task (new or traditional), two one-item and two two-item training sets preceded the experimental series. For all tasks, the children were presented with increasingly longer sets beginning with length 1 and continuing until they failed to recall the memory items of all three sets at a particular length. Testing was terminated at this point. Each correctly recalled set counted as one third, and the thirds were added up to provide a span score (Kemps, De Rammelaere, \& Desmet, 2000; Smith \& Scholey, 1992). For example, the correct recall of all the sets of one, two, and three items, of two sets of four items, and of one set of five items resulted in a span of $4[(3+3+3+2+1) \times 1 / 3]$.

The four WM tasks were administered in the middle of the academic semester (March) in two sessions of $30 \mathrm{~min}$ each. In the first session, the children were presented with the reading span task and then the reading letter span task. In the second session, the operation span and the continuous operation span tasks were presented, in that order.

\section{RESULTS}

\section{Descriptive Statistics}

The mean scholastic scores $(71 \%, S D=17 ; 73 \%$, $S D=17$; and $70 \%, S D=18$ of correct responses for the global, literacy, and mathematics scales, respectively) were close to the mean national scores observed in comparable middle schools (referenced as "collèges publics hors ZEP/REP" on the Web pages of the French Ministry of Education; $68 \%, S D=19 ; 68 \%, S D=17$; and $67 \%$, $S D=20$ for global, literacy, and mathematics scales, respectively), although our sample was slightly above the national mean. The mean spans from the new WM tasks (2.81, $S D=0.74$ and $2.32, S D=0.84$ for the reading letter and the continuous operation spans, respectively) did not significantly differ from the traditional mean spans $(2.77, S D=0.85$ and $2.46, S D=0.92$ for the reading and the operation spans, respectively). There was no tradeoff between spans and verification performance in the traditional span tasks $(r=.02, p=.86$ for the reading span task and $r=.11, p=.30$ for the operation span task). We computed the internal consistency reliability for each WM span task by considering the series to be recalled as items scored as either 1 or 0 , for correct or incorrect recall, respectively. Cronbach's (1951) alpha coefficients were .65 and .70 for the reading and operation spans, respectively, in comparison with .59 and .65 for the reading letter and continuous operation spans, respectively. The internal consistency reliability was thus almost equivalent for the four tasks.

\section{Correlation Analyses}

All of the WM spans were highly correlated with each other, particularly the new and traditional spans (see Table 1). These correlations were higher when the processing components of both new and traditional tasks were from the same domain $(r=.551$ between the reading and the reading letter spans, $r=.678$ between the operation and the continuous operation spans), but they reached a high level of significance even when the new and traditional tasks concerned different domains $(r=$ .492 between the reading and the continuous operation spans, $r=.460$ between the operation and the reading letter spans). These first results suggest that both types of span task aid in the evaluation of a common construct plus some specific capabilities related to the type of processing component. A principal component analysis confirmed the existence of a factor for the commonality in all four tasks, which accounted for $66 \%$ of the total variance, as well as a second factor that distinguished between "reading" and "arithmetic operation" tasks $(15 \%$ of the variance) and a third that distinguished between new and traditional tasks (11.5\% of the variance; see Table 2$)$.

More important, and as previously reported in the literature, both traditional spans were significantly correlated with the literacy score $(r \mathrm{~s}=.296$ and .316 for the reading and the operation spans, respectively), the mathematics score $(r \mathrm{~s}=.327$ and .353$)$, and the global score $(r \mathrm{~s}=.335$ and .359$)$. The compound traditional WM score obtained by averaging $z$ scores on the reading and

Table 1

Correlations Between Key Measures of WM Spans and Scholastic Performance

\begin{tabular}{lcccccccc}
\hline \multicolumn{1}{c}{ Key Measures } & 1 & 2 & 3 & 4 & 5 & 6 & 7 & 8 \\
\hline 1. Traditional compound span & - & & & & & & & \\
2. New compound span & .709 & - & & & & & & \\
3. Reading span & .900 & .610 & - & & & & & \\
4. Operation span & .900 & .625 & .621 & - & & & & \\
5. Reading letter span & .562 & .855 & .551 & .460 & - & & & \\
6. Continuous operation span & .650 & .855 & .492 & .678 & .461 & - & & \\
7. Literacy score & .341 & .497 & .296 & .316 & .462 & .385 & - & \\
8. Mathematics score & .377 & .521 & .327 & .353 & .479 & .411 & .811 & - \\
9. Global scholastic score & .385 & .538 & .335 & .359 & .499 & .421 & .947 & .957 \\
\hline
\end{tabular}

Note-Correlations $\geq .338$ are significant at $p=.001$, and correlations $\geq .267$ are significant at $p=.01$. 
Table 2

Factor Loadings for the Principal Component Analysis Performed on the Four WM Span Tasks

\begin{tabular}{lccr}
\hline \multirow{2}{*}{\multicolumn{1}{c}{ WM Span Task }} & \multicolumn{3}{c}{ Factors } \\
\cline { 2 - 4 } \multicolumn{1}{c}{1} & 2 & 3 \\
\hline Reading span & .823 & .205 & -.483 \\
Reading letter span & .748 & .568 & .330 \\
Operation span & .859 & -.313 & -.129 \\
Continuous operation span & .814 & -.399 & .321 \\
Variance & .659 & .155 & .115 \\
\hline
\end{tabular}

the operation spans correlated with the global scholastic score at $.385(p<.001)$. However, the spans given by the new WM tasks were even better predictors of academic performance. The reading letter span was a better predictor than the reading span $(r \mathrm{~s}=.462, .479$, and .499 vs. $.296, .327$, and .335 for the literacy, mathematics, and global scores, respectively), and the continuous operation span was a better predictor than the operation span $(r \mathrm{~s}=.385, .411$, and .421 vs. $.316, .353$, and .359 , respectively). The compound new WM score reached a significantly higher correlation with the global scholastic measure $(r=.538, p<.001)$ than did the compound traditional WM score $[r=.385, t(90)=2.25, p<.05]$. We therefore performed stepwise regression analyses to evaluate the extent to which new and traditional WM spans accounted for a shared variance.

\section{Regression Analyses}

In order to determine the predictive power of the two types of WM tasks (traditional vs. new), stepwise regression analyses were conducted on the literacy, mathematics, and global scholastic scores with the two compound WM scores as independent variables. The compound new WM score was the best predictor of the global scholastic score $\left[F(2,90)=37.12, R^{2}=.29, p<.0001\right]$, of the literacy score $\left[F(2,90)=30.93, R^{2}=.25, p<.0001\right]$, and of the mathematics score $\left[F(2,90)=33.85, R^{2}=.27\right.$, $p<.0001]$. The part of residual variance accounted for by the compound traditional WM score never reached significance $(F \mathrm{~s}<1)$, the highest $\Delta R^{2}$ value observed being .0001 . Thus, it appears that traditional WM tasks contributed no variance in addition to that already con- tributed by the new tasks. By contrast, forced regressions, in which the compound new WM score was entered after the compound traditional WM score, revealed that the new tasks still accounted for a substantial part of the remaining variance of the literacy score $[F(2,90)=$ 15.38, $\left.\Delta R^{2}=.13, p<.0005\right]$, the mathematics score $\left[F(2,90)=15.92, \Delta R^{2}=.13, p<.0005\right]$, and the global scholastic score $\left[F(2,90)=17.92, \Delta R^{2}=.14\right.$, $p<.0001$; see Table 3]. These results clearly indicate that the new computer-paced tasks account for an additional part of variance that is not accounted for by the traditional spans.

Detailed forward stepwise regression analyses were also conducted on the subcomponents of the scholastic scale with the four WM spans as independent variables. For each of these subcomponents, a significant contribution was never observed for traditional spans that were systematically preceded by the new spans. The reading letter span appeared in the first step for language comprehension, language tools, geometry, number knowledge and writing of numbers, and arithmetic problem solving, whereas the continuous operation span was the best predictor for written production, word problem solving, and comprehension of mathematical tools and concepts. The literacy, mathematics, and global scores were accounted for best by the reading letter $\operatorname{span}\left[R^{2} \mathrm{~s}=\right.$ $.22, .23$, and .25 , respectively; $p$ s $<.001$ ], the continuous operation span contributing in each case to a significant part of the remaining variance $\left[\varnothing R^{2} \mathrm{~s}=.04, .05\right.$, and .05 , respectively; $p s<.05]$. None of the traditional tasks contributed significantly to the residual variance.

\section{DISCUSSION}

These results clearly indicate that, in comparison with traditional tasks, the new WM span tasks designed by Barrouillet et al. (2004) provide a more accurate evaluation of the capacity of the common mechanism reflected by WM span measures. This finding is all the more remarkable when it is considered that those tasks that involve the simplest activities and hamper the planning of sophisticated dual-task strategies turned out to be the most predictive of complex cognitive achievements. The predictive power of the new WM span tasks echoes pre-

Table 3

Stepwise Regressions for Scholastic Performance

\begin{tabular}{clllllll}
\hline \multirow{2}{*}{ Dependent Variable } & \multicolumn{3}{c}{ Forward } & & \multicolumn{3}{c}{ Forced } \\
\cline { 2 - 5 } \cline { 6 - 8 } & Factor & Step & $\Delta R^{2}$ & & Factor & Step & $\Delta R^{2}$ \\
\hline Literacy & New WM & 1 & $.25^{*}$ & & Traditional WM & 1 & $.13^{*}$ \\
& Traditional WM & 2 & .00 & & New WM & 2 & $.13^{*}$ \\
Mathematics & New WM & 1 & $.27^{*}$ & & Traditional WM & 1 & $.14^{*}$ \\
& Traditional WM & 2 & .00 & & New WM & 2 & $.13^{*}$ \\
& New WM & 1 & $.29^{*}$ & & Traditional WM & 1 & $.15^{*}$ \\
& Traditional WM & 2 & .00 & & New WM & 2 & $.14^{*}$ \\
\hline
\end{tabular}

Note- "New WM" and "traditional WM" refer to the compound new and compound traditional WM scores, respectively. ${ }^{*} p<.05$. 
vious observations by Fry and Hale (1996), who observed that performance on WM tasks as simple as reporting the colors of items while maintaining their identities or locations in view of their subsequent recall was highly correlated with fluid intelligence. A first conclusion, therefore, is that the complexity of the processing component in most of the traditional WM span tasks (reading span, operation span, alphabet recoding, and $A B C D)$ is a superfluous characteristic. In fact, selfpaced WM span tasks require complex activities to induce the necessary time pressure that is inherent to their structure. The second conclusion is that the predictive value of the traditional WM spans does not stem from their capacity to assess an ability to strategically cope with the demands of complex span tasks - which would also be involved in any complex activity-because when the possibility of dealing strategically with the task is reduced by computer-paced presentation, the predictive value is increased. This does not mean that mnemonic strategies for encoding and maintaining memory items are unimportant in complex spans (McNamara \& Scott, 2001). However, our results show that complexity and strategies do not contribute to the predictive value of WM spans but rather introduce more noise than information into their relationship with high-level cognition. This conclusion has theoretical and practical implications.

The predictive power of span tasks with simple processing components suggests that WM tasks measure some fundamental and general capacity involved in both elementary and complex cognitive processing. This result echoes models of WM that conceive of cognitive resources as a kind of mental energy required to produce activation (Engle, Cantor, \& Carullo, 1992; Just \& Carpenter, 1992; Lovett, Reder, \& Lebière, 1999). This result is also in line with the time-based resource-sharing model (Barrouillet et al., 2004), which assumes, following Anderson (1993) and Cowan (1995), that this fundamental capacity is attentional. This attention is required for retrieval of knowledge from long-term memory during reading of letters or solution of simple operations, but also for reactivation of the decaying memory traces of the items to be recalled. The time constraints that result from the computer-paced presentation imply that even simple secondary tasks almost continuously capture attention and impede the refreshing of memory traces. Thus, the ability of individuals to prevent the decay of these traces reflects their available amount of attention - that is, their WM capacity. By contrast, the predictive power of the new tasks is at odds with theories of WM such as that of Ericsson and Kintsch (1995), in which any notion of cognitive resources is denied and by which it is assumed that the relationship between WM and complex cognition is due to strategic factors and knowledge. Indeed, when discussing Just and Carpenter's (1992) capacity theory of comprehension, Ericsson and Kintsch assumed that their proposal for long-term WM "can do without the somewhat slippery notion of cognitive resources altogether" but rather "emphasizes good readers' use of more sophisticated, more complex comprehension strategies that result in the generation of more extensive retrieval structures and hence a larger effective WM" (Ericsson \& Kintsch, 1995, p. 229). Moreover, the present results strengthen and extend the theoretical interest of the WM construct by going beyond the rather circular observation that performance in span tasks that involve complex processes (e.g., reading sentences while remembering words) is correlated with performance in high-level cognition (e.g., reading comprehension). The results demonstrate that WM span tasks measure a fundamental capacity and that WM measures are central to any assessment of cognitive abilities. Computerpaced WM span tasks could thus constitute useful tools. Apart from being fast and easy to administer, they permit better control of the strategies that undermine the rationale of WM span tasks. Moreover, they do not suffer from the undesirable effect of large individual differences in complex skills. Individuals can differ greatly in literacy and mathematics skills, but reading letters and browsing the number chain are universal skills in literate groups even in rather young children. The range of tasks that permit a valid measure of the WM capacity is thus considerably extended.

\section{REFERENCES}

Anderson, J. R. (1993). Rules of the mind. Hillsdale, NJ: Erlbaum. BADDELEY, A. D. (1990). Human memory: Theory and practice. Hillsdale, NJ: Erlbaum.

Baddeley, A. D., \& Hitch, G. J. (1974). Working memory. In G. A. Bower (Ed.), Recent advances in learning and motivation (Vol. 8, pp. 647-667). New York: Academic Press.

Baddeley, A. D., Logie, R., Nimmo-Smith, I., \& Brereton, N. (1985). Components of fluent reading. Journal of Memory \& Language, 24, 119-131.

BARROUILLET, P. (1996). Transitive inferences from set-inclusion relations and working memory. Journal of Experimental Psychology: Learning, Memory, \& Cognition, 22, 1408-1422.

Barrouillet, P., Bernardin, S., \& CAmos, V. (2004). Time constraints and resource sharing in adults' working memory spans. Journal of Experimental Psychology: General, 133, 83-100.

Case, R., Kurland, M., \& Goldberg, J. (1982). Operational efficiency and the growth of short-term memory. Journal of Experimental Child Psychology, 33, 386-404.

CowAN, N. (1995). Attention and memory: An integrated framework. New York: Oxford University Press.

Cronbach, L. J. (1951). Coefficient alpha and the internal structure of tests. Psychometrika, 16, 297-334.

DANEMAN, M., \& CARPENTER, P. A. (1980). Individual differences in working memory and reading. Journal of Verbal Learning \& Verbal Behavior, 19, 450-466.

DANEMAN, M., \& MERIKLE, P. M. (1996). Working memory and comprehension: A meta-analysis. Psychonomic Bulletin \& Review, 3, 422-433.

Engle, R. W., Cantor, J., \& Carullo, J. J. (1992). Individual differences in working memory and comprehension: A test of four hypotheses. Journal of Experimental Psychology: Learning, Memory, \& Cognition, 5, 972-992.

Engle, R. W., Kane, M. J., \& Tuholski, S. W. (1999). Individual differences in working memory capacity and what they tell us about controlled attention, general fluid intelligence, and functions of the prefrontal cortex. In A. Miyake \& P. Shah (Eds.), Models of working memory: Mechanisms of active maintenance and executive control (pp. 102-134). Cambridge: Cambridge University Press.

Engle, R. W., Tuholski, S. W., Laughlin, J. E., \& Conway, A. R. 
(1999). Working memory, short term memory, and general fluid intelligence: A latent variable approach. Journal of Experimental Psychology: General, 128, 309-331.

ERICSSON, K. A., \& KINTSCH, W. (1995). Long-term working memory. Psychological Review, 102, 211-245.

FrY, A. F., \& HALE, S. (1996). Processing speed, working memory, and fluid intelligence: Evidence for a developmental cascade. Psychological Science, 7,237-241.

JuST, M. A., \& CARPENTER, P. A. (1992). A capacity theory of comprehension: Individual differences in working memory. Psychological Review, 99, 122-149.

Kemps, E., De Rammelaere, S., \& Desmet, T. (2000). The development of working memory: Exploring the complementarity of two models. Journal of Experimental Child Psychology, 77, 89-109.

Kyllonen, P. C., \& Christal, R. E. (1990). Reasoning ability is (little more than) working-memory capacity? Intelligence, 33, 1-64.

Lovett, M. C., Reder, L. M., \& Lebière, C. (1999). Modeling working memory in a unified architecture: An ACT-R perspective. In A. Miyake \& P. Shah (Eds.), Models of working memory: Mechanisms of active maintenance and executive control (pp. 135-182). Cambridge: Cambridge University Press.

McNamara, D. S., \& ScotT, J. L. (2001). Working memory capacity and strategy use. Memory \& Cognition, 29, 10-17.

SHute, V. J. (1991). Who is likely to acquire programming skills? Journal of Educational Computing Research, 7, 1-24.
Smith, M. M., \& Scholey, K. A. (1992). Determining spatial span: The role of movement time and articulation rate. Quarterly Journal of Experimental Psychology, 45A, 479-501.

Turner, M. L., \& Engle, R. W. (1989). Is working memory taskdependent? Journal of Memory \& Language, 28, 127-154.

\section{NOTES}

1. By traditional self-paced WM span tasks, we do not intend to refer to self-presented tasks in which the entire procedure is driven by the participant, but to tasks in which the participant performs the processing component at his or her own pace. In some traditional tasks, such as Daneman and Carpenter's (1980) reading span or Case et al.'s (1982) counting span, it is not the participant but the experimenter who displays the next sentence to be read or the next array to be enumerated. However, these tasks remain self-paced in that there is no time constraint in reading the sentence or in counting the array, the participant being free to perform these activities at his or her own pace.

2. The definitions of these subdomains by the French Ministry of Education and the national results can be found on the Web at http:// evace26.education.gouv.fr/.

(Manuscript received December 11, 2003; revision accepted for publication March 23, 2004.) 